\title{
Références
}

\section{Les compétences de l'enseignant clinicien et le modèle de rôle en formation clinique}

\author{
Martine CHAMBELAND*, René HIVON*
}

\begin{abstract}
Messages clés
- Les organismesd'agrément des programmes de formation médicale pré et post-doctoraux, en réponseaux demandessocié tales, exigent dorénavant un enseignement systématique del'ensemble des dimensions de la compétence professionnelle, telles que le raisonnement clinique, la communication, l'éhique, le travail collaboratif et le professionnalisme - Ces nouvelles exigences imposent, d'une part, la mise en cauvre d'activités d'enseignement formel dans lescursus; ellesappellent, en outre, de façon privilégiée, une réflexion approfondie concernant la longue période de la formation dinique - L e plus souvent, l'enseignement dinique de l'ensemble de ces dimensions est informe, aléatoire et implicite; les enseignants cliniciens ont peu ou pas de préparation pour l'exercice des deux modalités pédagogiques spécifiques à l'enseignement clinique que sont le modèle de rôle et la supervision. • L'importance détermi nante du modèle de rôle pour l'apprentissage des éudiants fait consensus et les caractéristiques des bons modèles de rôle sont connues - Certaines d'entre elles peuvent être acquises ou modifiées et pourrai ent éventuellement faire l'objet de perfectionnement pédagogique pour les enseignants cliniciens.
\end{abstract}

\section{Mots clés}

Enseignement clinique; compétence professionnelle; enseignant clinicien ; modèle de rôle.

\section{Key Messages}

- In response to societal needs, accreditation agencies for medical training now require systematic teaching, within undergraduate and graduate medical programs, of all the dimensions of professional competence, including clinical reasoning, communication skills, ethics, team work and professionalism. - While medical curricula mus include formal teaching and learning activities for basc knowledge relevant to these dimensions, these new requirements will affect the entire period of dinical education in a significant way. - Clinical teaching, performed in the context of real medical practice is most often informal, random and implicit; clinical teachers receivelittle formal preparation regarding the two specific teaching modalities of clinical teaching, namely role modeling and dinical supervision. - The significant impact of dinical teachers as role models on students' learning is well recognized and the characteristics of excellent role models have been des cribed. - Some of these characteri stics could be acquired or modi fied and this could form the basis for future faculty deve lopment for clinical teachers

\section{Key words \\ Clinical teaching; professional competence; clinical teacher; role modes; role modeling.}

Pédagogie M édicale 2005; 6: 98-111

*Centre de Pédagogie des Sciences de la Santé- Facultéde Médecine- Universitéde Sherbrooke- Sherbrooke- Québec, Canada Correspondance : Martine Chamberland - Centre de Pédagogie des Sciences de la Santé - Faculté de Médecine - Université de Sherbrooke - $300112^{\circ}$ avenue Nord - Sherbrooke - Québec, Canada, J1H 5N4 - Téléphone : (1) 819-564-5385 Mailto: martine.chamberland@usherbrooke.ca 


\section{Introduction}

D epuis ces dernières années, les facultés de médecine doivent répondre à de nouvelles exigences pour leurs programmes de formation ainsi que pour la qualité des futurs diplômés. Les tranformations du contexte social, les modifications de la pratique médicale et l'exigence accrue d'une responsabilité sociale des universités fondent la réflexion des organismes d'agrément à propos des caracté ristiques souhaitées pour les médecins du nouveau millé naire. Cette réflexion et ce mouvement, qui sont non seulement québécois et canadiens mais aussi nord-américains et européens, ont conduit à la publication de nombreux rapports qui déterminent dorénavant les normes d'agrément pour les facultés de médecine : le «Rapport de la commission de la médecine des années 2000 du Collège des M édecins du Q uébec »"; le "CanM ED S 2000 » du Collège Royal des médecins et chirurgiensdu Canada ${ }^{2}$; le "Graduate Medical Education Core Curriculum » de I'American Association of Medical Colleges ${ }^{3}$; le «T omorrow's D octors » du General M edical Council ${ }^{4}$. Les recommandations provenant de ces différentes sources, bien que rédigées avec des terminologies différentes, sont convergentes. Elles décrivent les caractéristiques des futurs diplômés qui vont largement au-delà de l'acquisition et de I'application technique des connaissances biomédicales en y ajoutant defaçon explicite d'autres dimensionstelles que la communication, l'éthique, le travail collaboratif, l'apprentissage efficace continu et le professionnalisme.

Bien que ces nouvelles exigences imposent la miseen place d'enseignements formels et spécifiques dans les cursus, elles appellent surtout, selon nous, une réflexion approfondie concernant la formation clinique. C'est durant cette partie de la formation, qui s'étend sur plusieurs années, que l'ensemble des dimensions de la compétence professionnelle sont graduellement développées et mises en action quotidiennement. II importe donc de porter une attention particulière aux contenus et à la manière dont se fait l'enseignement au cours de la formation clinique.

Actuellement, de façon générale, l'enseignement clinique réal isé dans le cadre des activités de soins est globalement informe, implicite et aléatoire. II est rarement intentionnel et planifié et il est à la merci des aléas des soins et de l'exposition clinique des étudiants. Les enseignants cliniciens intègrent automatiquement l'ensemble des caractéristiques de la compétence professionnelle dans leur pratique quotidienne de clinicien. Pour quider les étudiants dans leurs apprentissages, ils font appel à leur savoir pratique de bons cliniciens. Le raisonnement clinique, au cœur de la pratique médicale et très proche du savoir biomédical, est de fait un peu plus facile à expliciter de la part des enseignants cliniciens ; cependant, les compétences intégrées et les savoir-faire complexes qu'ils ont développés, en particulier dans les champs de la communication, de l'éthique et du professionnalisme, sont et demeurent largement tacites, difficilement accessibles aux étudiants. Les conséquences d'un tel enseignement clinique, largement implicite, informel et aléatoire, sur l'apprentissage des externes et des résidents sont rarement appréhendées de façon systématique. À notre connaissance, il n'y a pas d'étude spécifique sur ce sujet. Cependant, les observations des professeurs, des instances facultaires et de l'ensemble des organismes concernés par l'éducation médicale, qui sont à la base des nouvelles normes de formation, vont dans le même sens : les futurs médecins devraient être mieux formés aux dimensions de leur compétence professionnelle qui ont été récemment explicitées telles que la communication, l'éthique, le travail collaboratif et le professionnalisme. De plus, la prépondérance des problèmes de communication, identifiée dans le cadre des plaintes déposées contre les médecins, est vraisemblablement un indice qu'il y a une nécessité d'améliorer l'enseignement de cette dimension. Par ailleurs, $\mathrm{H}$ afferty et Franks $5^{5,6}$ ont attiré l'attention des éducateurs médicaux sur l'apprentissage de l'éthique fait par les étudiants en médecine et sur l'influence de chacune des composantes del'environnement pédagogique À leur avis, l'environnement pédagogique déborde largement le curriculum formel (objectifs, contenus, etc.). Le curriculum informel, particulièrement en cause au cours de l'enseignement clinique (modèle de rôle, supervision), qui situe l'apprentissage au niveau des interactions interpersonnelles, et le curriculum caché (hidden curriculum), véhiculé par les structures organisationnelles et décisionnelles de l'institution, sont deux autres éléments de l'environnement pédagogique qui exercent une influence encore plus déterminante dans l'apprentissage des rôles professionnels des étudiants; malheureusement, ils ne le font pas toujours dans la direction souhaitée La figure 1 illustre les interrelations possibles entre ces trois curricula et leurs influences sur l'apprentissage.

Dès lors que la communication et l'éthique, tout comme le raisonnement clinique et les gestes techniques, ne sont plus perçus comme des habiletés innées ou comme des dimensions personnelles mais plutôt comme des compé tences professionnelles à acquérir et à évaluer lors de la 


\section{Références}

formation, il devient obligatoire d'envisager un enseigne ment, un apprentissage et uneévaluation davantage structurés. Ceci semble désormais faire l'objet d'un large consensus.

Paradoxalement, malgrél'importanceque l'on doive doré navant accorder à la formation clinique et aux enseignementsqui y sont dispensés, la préparation pédagogique du corps professoral à cette tâche reçoit quant à elle bien peu d'attention. II semble exister certaines croyances de la part des professeurs et des facultés à l'égard de l'enseignement clinique, qui sous-estiment sa complexité réelle. Certains prétendent que l'enseignement clinique, puisqu'il est très intriqué à la pratique clinique, se fait spontanément et avec un minimum de préparation. L'« osmose » est pour ceux-ci une modalité pédagogique privilégiée, comme en témoigne cet énoncé célèbre en enseignement clinique : "s see one, do one, teach one».

C'est avec l'intention de préparer de façon optimale les professeurs au défi actuel de l'enseignement clinique, qui est celui intégrer de façon explicite l'ensemble des dimensions de la compétence professionnelle, que nous avons entrepris notre réflexion. Avant de pouvoir développer des activités de perfectionnement pédagogique appropriées, il fallait préalablement répondre aux deux questions suivantes : quelles sont les compétences du professeur clinicien expert? Q uelles sont les modalités pédagogiques à privilégier dans le cadre de la formation clinique?

Dans cet article, nous proposons une réponse possible à ces questions, qui sinspire à la fois de nos réflexions et d'une recension des écrits pertinents sur le sujet. Cette recension des écritss'est faiteà partir de la banque de données MEDLINE, de la révision bibliographique des articles importants retrouvés et de la révision manuelle de certains périodiques spécialisés en éducation médicale mais non indexés jusqu'à une date récente (Teaching and Learning in Medicine, Medical Teacher). La recherche couvre la période 1993-2004 et a utilisé les mots-clés suivants: enseignant clinicien (dinical teacher), enseignement clinique (clinical teaching), modèle de rôle (role mode, role modeling) ; perfectionnement pédagogique (faculty deve lopment).

$\mathrm{N}$ ous présenterons notre réponse à ces questions en abordant successivement les 3 thèmes suivants : 1) les compé tences du professeur clinicien expert ; 2) le contexte de l'enseignement clinique et ses modalités spécifiques ; 3) le modèle de rôle.

Figure 1 :

Les trois curricula et leurs influences sur l'apprentissage

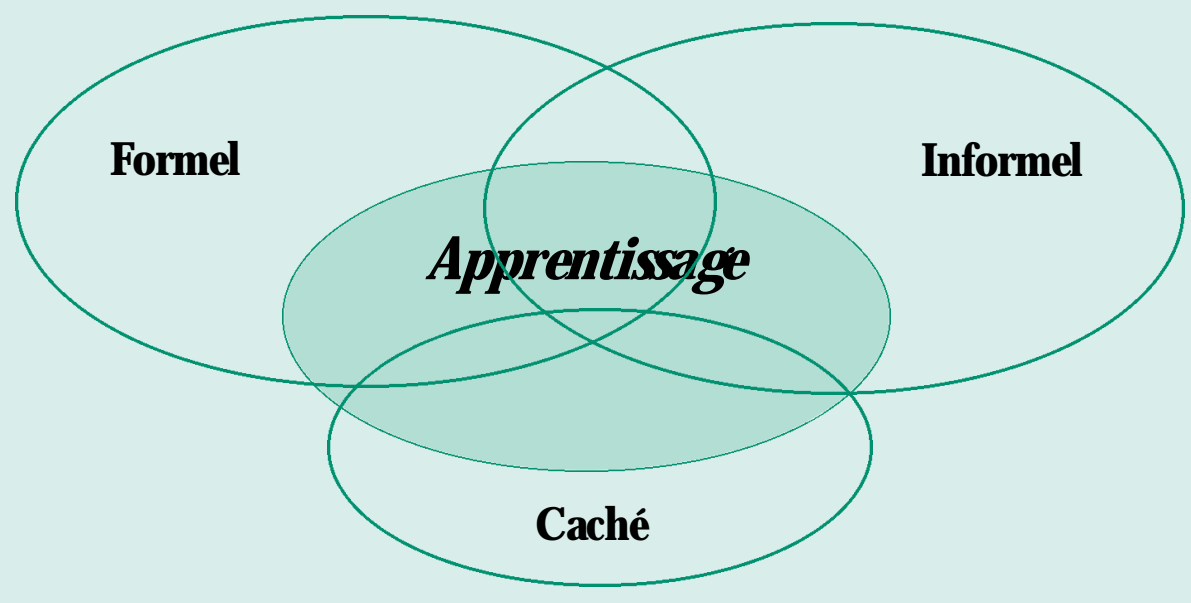

L'environnement pédagogi que comprend trois curricula : leforme, l'informe e le curriculum caché Cette figureillustre les interrelations possibles entre ces trois curricula et leurs influences sur l'apprentissage. 


\section{Les compétences de l'enseignant dinicien expert}

Le perfectionnement pédagogique quel'on veut mettreen place vise à améliorer et à développer les compétences des professeurs cliniciens en enseignement clinique. Q ue saiton de ces compétences? Comment la recherche actuelle décrit-elle l'enseignant clinicien expert? N ous trouvons deux types de données dans les écrits recensés. D 'une part, plusieurs auteurs proposent des synthèses et des réflexions sur le sujet ${ }^{7-11}$. D'autre part, Irby ${ }^{12-14}$ a publié une série d'études qualitatives visant à décrire les types de connaissances, les stratégies ou le raisonnement pédagogique d'excellents enseignants cliniciens. L'ensemble de ces sources converge vers la coexistence nécessaire d'une double compétence : la compétence clinique et la compé tence pédagogique.

\section{La compétence clinique}

Selon Prideaux et al. ${ }^{8}$ " good dinical practice is at the heart of good clinical teaching ». Puisque les compétences médicales constituent les « contenus » spécifiques à enseigner par l'enseignant clinicien, celui-ci doit êtrelui-même, avant tout, un clinicien expert $7,12,15,16$. Selon la conception que proposent $\mathrm{H}$ iggs et Jones ${ }^{17}$, l'expertise clinique est multidimensionnelle. Au centre de cette expertise, on trouve le processus de résolution de problèmes de santé des patients - le raisonnement clinique -, auquel sintègrent et contribuent les connaissances spécifiques, les habiletés cliniques et techniques, le jugement professionnel, la communication et les habiletés interpersonnelles et les stratégies métacognitives. Cela signifie que l'enseignant clinicien doit avoir développé et intégré les connaissances relatives à l'ensemble des compétences et qu'il doit les appliquer efficacement dans sa pratique clinique quotidienne.

Le clinicien, dans un contexte de formation, doit être particulièrement métacognitif, c'est-à-dire qu'il doit être explicite par rapport à sa propre expertise professionnelle et à son exercice quotidien ${ }^{18}$. II doit respectivement prendre conscience de ses connaissances et de ses actions, être capable d'expliciter, d'articuler, d'expliquer, de critiquer voire de corriger ses actions et, in fine, être en mesure de rendre visible et accessible à l'apprentissage non seulement le résultat de sa démarche de solution de problème mais surtout son processus ainsi que l'articulation des savoirs sous-jacents 8 . Cette nécessaire explicitation de la part de l'enseignant n'est pas toujours facile à réaliser pour le clinicien expert puisque, paradoxalement, la rapidité d'exécution et l'automatisation des connaissances d'action sont également des caractéristiques de l'expertise médical $\mathrm{e}^{18}$.

\section{La compétence pédagogique}

Au fil des années, comme dans les autres domaines de la pédagogie universitaire, la croyance quel'expertise disciplinaire, en l'occurrence clinique, était une condition suffisante pour enseigner adéquatement la médecine a cédé graduellement le pasà l'évidence dela nécessitéd'une compétence supplémentaire dans un champ disciplinaire dis tinct, celui de la pédagogie Les travaux de Irby dans les années 90 ont été marquants à cet égard. En utilisant des analyses de cas et des méthodes qualitatives, il s'est préoccupé de décrire minutieusement le fonctionnement d'excellents enseignants cliniciens en médecine interne dans le contexte de l'enseignement clinique, en particulier lors des tournées d'enseignement pendant des stages à l'hôpital 14, 19. Inspiré des modèles d'organisation de connaissances de Shulman ${ }^{20,21}$ pour l'enseignant expert et de Schmidt et al. ${ }^{22}$ pour le clinicien expert, Irby a décrit les différents domaines de connaissances qu'utilisent et qu'articulent ces enseignants cliniciens experts en contexte de formation. Aux domaines de connaissances relatifs à la médecine (les connaissances médicales, les patients, les contextes cliniques) sajoutent des domaines relatifsà l'éducation (les principes d'enseignement et d'apprentissage, les caractéristiques des apprenants, etc.). A l'interface de ces domaines distincts, les enseignants experts ont également développéun champ deconnaissances d'action spécifiques, celui des scénarios d'enseignement (« teaching scripts»), qui informe sur la manière d'enseigner de façon ciblée et efficace des connaissances spécifiques pour un niveau donné d'apprenants ${ }^{12}$.

Irby ${ }^{14}$ a de plus décrit comment ces mêmes enseignants, pendant la formation clinique, sont engagés simultané ment avec leurs patients et les étudiants dans un double processus de raisonnement clinique et de raisonnement pédagogique. $D$ ans cette étude, il démontre que les enseignants cliniciens : planifient l'enseignement au préalable, diagnostiquent le niveau de connaissances et de compré hension des étudiants en les écoutant et en les questionnant, prennent des décisions pédagogiques adaptées aux étudiants et aux situations cliniques disponibles. Irby ${ }^{14}$ et Pinsky et al. ${ }^{23,24}$ ont également mis en évidence comment 


\section{Références}

les excellents enseignants cliniciens utilisent des stratégies métacognitives, telles que la planification et l'évaluation, par la réflexion dans l'action et après l'action, pour amé liorer leur enseignement.

La complexité de l'enseignement clinique, le caractère multidimensionnel des connaissances nécessaires à son exercice, la démonstration qu'il s'agit d'un processus de résolution de problèmes qui exige une pratique réflexive très apparentéeà celle décritepar Schön ${ }^{25}$ témoignent clairement que l'enseignement clinique appartient au domaine de la pratique professionnelle. $\mathrm{H}_{\text {esketh et son équipe }}{ }^{7}$ ont également établi récemment un parallèle étroit entre l'expertise clinique et l'expertise en enseignement, en proposant une définition holistique des rôles de l'enseignant clinicien qui utilise une approche prenant en compte les conséquences de ses actions ( « outcome-based » education), de façon similaire à celle utilisée pour définir l'expertise clinique $^{26}$. Avant de discuter davantage les modalités d'interventions pédagogiques propres à l'enseignement clinique, il convient d'abord de caractériser le contexte particulier dans lequel s'effectue cet enseignement, pour mettre en évidence sa complexité, ses contraintes et son potentiel extraordinaire.

\section{Le contexte de l'enseignement clinique et ses modalités pédagogiques spécifiques}

La tâche d'enseignant est complexe et difficile; cependant, dans l'univers de l'enseignement médical, l'enseignement clinique se démarque par plusieurs exigences et défis qu'il pose quotidiennement aux enseignants clinicien $\mathrm{S}^{27}$ : 1) I'objectif ultime de cet enseignement - la formation de praticiens compétents - , confère une responsabilité particulière aux enseignants ; 2) ceux-ci doivent concilier au même moment les tâches de soins et d'enseignement et sengager ainsi simultanément dans un double processus de résolution de problème: le rai sonnement clinique pour les soins des patients et le rai sonnement pédagogique pour l'enseignement et l'apprentissage des externes et des résidents ${ }^{12,14} ; 3$ ) lecontenu même de l'enseignement clinique - les compétences médicales -, exige de la part des enseignants cliniciens qu'ils abordent et qu'ils articulent de multiples dimensions de façon concomitante; 4) les enseignants travaillent simultanément avec une vaste hétérogénété d'apprenants en termes de niveaux d'expérience et d'orientations disciplinaires, ils utilisent une variété de méthodes pédagogiques et ils évoluent dans différents contextes de soins (intra-hospitalier, soins ambulatoires,... ) ; 5) le contexte d'enseignement est celui de la pratique médicale réelle à l'intérieur de laquelle les enseignants n'exercent qu'un contrôle relatif sur certains élé ments pourtant déterminants pour l'enseignement et l'apprentissage, tels que le débit clinique, la variété des problèmes présentés par les patients et l'organisation du temps ${ }^{28}$. Toutes ces exigences et ces défis spécifiques du contexte dans lequel s'effectue l'enseignement clinique requièrent de l'enseignant clinicien qu'il sadapte aux conditions d'exercice clinique et pédagogique en mouvement continuel.

La dual ité des activités de soins et d'enseignement est souvent perçue comme conflictuelle. Pourtant, l'engagement dans la réalisation de tâches professionnelles authentiques constitue le contexte d'apprentissage potentiellement le plus motivant pour l'étudiant et le plus puissant pour l'enseignement et l'apprentissage des compéences inté grées au service de la résolution de problèmes de santé des patients. D eux perspectives éducatives théoriques, la théorie de l'apprentissage expérientiel 25, 29, 30 et l'approche de I'apprentissage contextualisé31, 32, nous permettent de mieux comprendre l'apprentissage et le développement des compétences dans le contexte de la pratique en formation clinique. Elles nous permettent également d'anticiper les rôles et les interventions éventuellement efficaces des enseignants cliniciens. La théorie de l'apprentissage expérientiel signale l'importance et la place centrale de l'expérience pratique dans le développement des compé tences. $M$ ais la pratique seule, bien que nécessaire, n'est pas suffisante et doit s'associer à de la réflexion pendant et après l'action pour permettre une interprétation et une éventuelle modification des schèmes conceptuels préexistants. Cette triade de contextualisation, décontextualisation (ou abstraction) et recontextualisation est d'ailleurs à la base du transfert des connaissance ${ }^{33}$. Le processus d'apprentissage actif dépend del'étudiant, mais le rôledu professeur est central en tant que modèle de rôle et de superviseur, pour susciter et promouvoir le processus de réflexion et pour valider ou corriger le résultat du processus $^{34}$. L'approche de l'apprentissage contextualisé met l'emphase, quant à elle, sur le contexte dans lequel l'apprentissage s'effectue. Selon cette perspective, les connaissances apprises sont indissociables de leur contexte d'apprentissage. En formation clinique, le contexte réd fournit non seulement une clé d'encodage performante pour le rappel ultérieur des connaissances mais également tout un ensemble d'éléments tacites et explicites de l'environ- 
nement social et fonctionnel propreà la pratique médicale. D ans cette même perspective, Lave et Wenger ${ }^{35}$, en développant leur modèle de " participation périphérique légitime » («legitimate peripheral participation »), repris par Pratt ${ }^{36}$ pour l'éducation supérieure, nous informent davantage sur le processus d'apprentissage et, de ce fait, sur le rôle possible des enseignants. Selon ce modèle, l'étudiant, même novice, participeinévitablement àune communautédepraticiens. Par l'observation des modèles, grâce à la pratique et à la supervision en situation authentique, il développe progressivement ses compétences et migre peu à peu d'un rôle initialement « périphérique » vers une position de plus en plus centrale comme praticien actif dans le milieu socioculturel de la profession médicale.

Ces perspectives permettent d'identifier les éléments déterminants de la formation clinique et leurs interactions. Ces éléments sont les patients, les enseignants cliniciens, les étudiants et le contexte de la pratique ; leurs interactions conditionnent à la fois les contenus et les apprentissages qui seront réalisés ${ }^{37}$. De la discussion pré cédente, découlent deux modalités d'interventions spécifiques du professeur en enseignement clinique. Ces deux modalités sont respectivement le modèle de rôle et la supervision. Elles sont spécifiques de l'enseignement clinique puisqu'eles mettent en interaction simultanément I'enseignant clinicien, le patient et l'étudiant, à la fois pour résoudre le problème du patient et pour contribuer à l'apprentissage de l'étudiant. La figure 2 illustre cette dynamique. En tant que modèle de rôle, l'enseignant clinicien est en exercice clinique réé et il est observé par l'étudiant. D ans le cadre de la supervision, il y a permutation des rôles : l'étudiant est alors directement en interaction avec le malade tandis quel'enseignant clinicien devient l'observateur et le guide.

La réforme de l'enseignement clinique qui sera nécessaire pour optimiser le développement professionnel multidimensionnel des futurs diplômés doit donc cibler les élé ments déterminants de cette période de formation ${ }^{37,38}$. D'ores et déjà, les éducateurs médicaux se sont intéressés à l'adéquation des contextes de soins et des conditions cliniques présentées par les patients et auxquels sont exposés les externes et les résidents. L'accroissement de la formation en contexte de soins ambulatoires et à l'extérieur de I'hôpital universitaire vers les milieux communautaires s'inscrit dans cette perspective. M ais, au-delà de ces changements déjà entamés, il faut maintenant investir

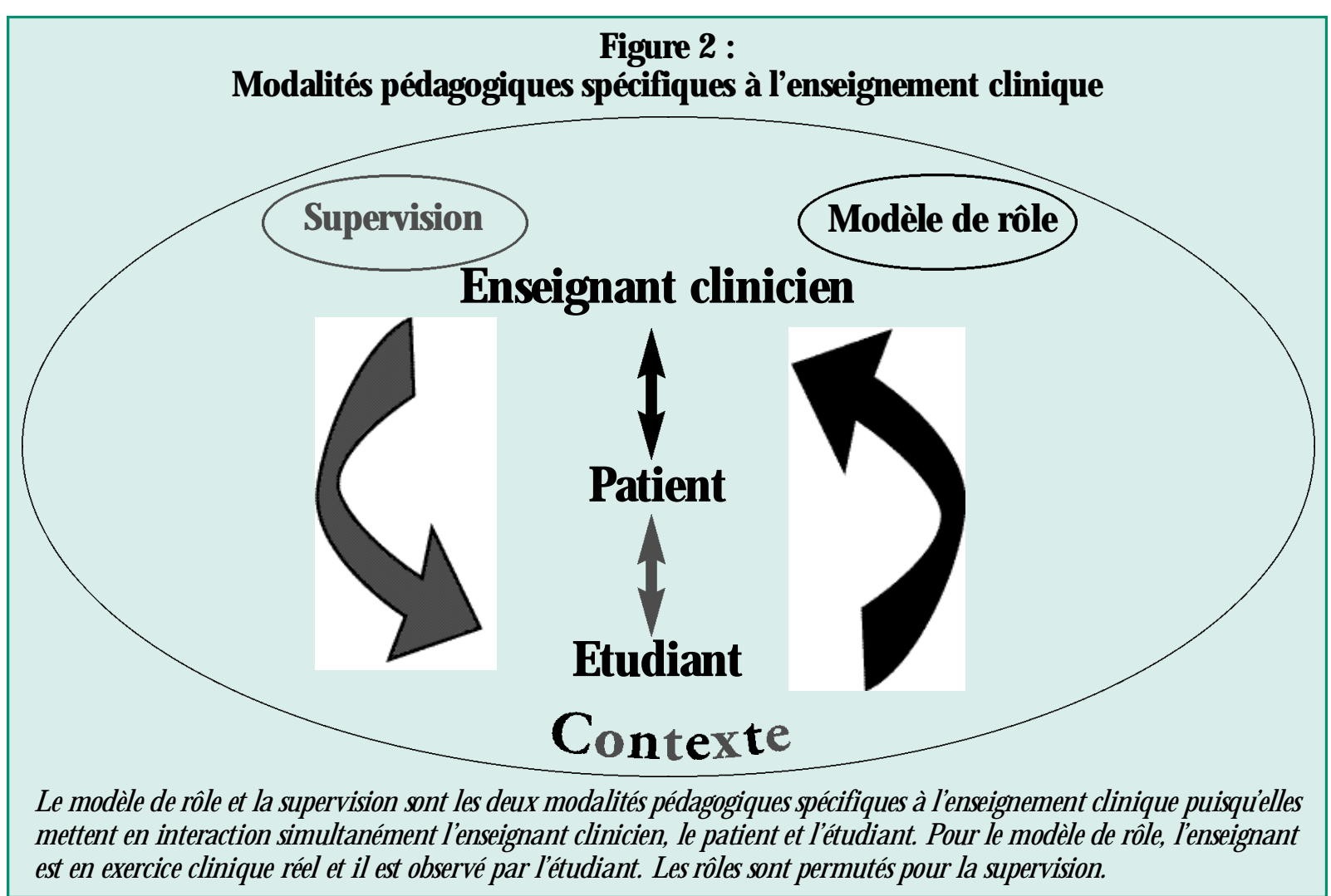




\section{Références}

davantage en direction des acteurs principaux et en particulier sintéresser aux enseignants et au modèle de rôle qu'ils exercent, élément crucial mais jusqu'à maintenant négligé dans les réformes de l'enseignement professionne ${ }^{39}$. C'est pourquoi il est utile d'exposer une synthèse plus approfondie des données de la recherche actuelle ment disponibles concernant le modèle de rôle

\section{Le modàle de rôle}

La recension des écrits concernant le modèle de rôle sera présentée autour des quatre éléments suivants : 1) l'importance et l'impact des modèles de rôle sur les étudiants; 2) la définition du modèle de rôle; 3 ) les caractéristiques des bons modèles de rôle et : 4) les limites actuelles des modèles de rôle N ous utilisonsleterme «modèlederôle» - au singulier - pour décrire la modalité pédagogique (en anglais: « role modeling »). Le terme «modèles de rôle »au pluriel - sera, quant à lui, utilisé pour décrire les individus, en l'occurrence les enseignants cliniciens, qui exercent le modèle de rôle (en anglais: « role models»).

\section{L'importance ou l'impact des modèles de rôle sur les étudiants}

Tosteson ${ }^{40}$ rapportait ceci: «When I ask an educated person : "What was the most significant experience in your education?" I almost never get back an idea but almost alwaysa person. M embers of professions and the mot obstruse scholars usually say», «lt was when I came to know Professor or D octor... »... «Redations with other persons drive our feelings and thus our actions I believe that the modern jargon is "role models"». Plus de vingt ans plustard, M audsley ${ }^{41}$ rapportait les propossuivants d'Albert Schweitzer qui marquent toute l'importance des modèles de rôle: «Exampleisnot themain thing in influencing others, it is the only things.

En considérant l'objet de l'apprentissageà réaliser pendant la formation clinique, on peut saisir l'importance et la contribution potentiellement unique des modèles de rôle. Les externes et les résidents doivent développer cette capacité de résoudre des problèmes complexes, uniques, flous, imprécis et incertains, qui commandent une intégration de l'ensemble des dimensions de la compétence professionnelle. L'ensemble de ces savoir-agir professionnels complexes échappe d'ailleurs à une codification systématique et aucun répertoire complet de toutes les situations cliniques possibles n'est disponible. II n'existe aucun ouvrage de référence ( « textbook ») capable d'illustrer l'ex- pertise clinique en action, la science et l'art de la médecine, comme peuvent le faire les cliniciens en exercice qu'observent quotidiennement les étudiants. II n'existe aucun ouvrage de référence du savoir tacite pourtant caractéristique de l'expert. Les enseignants cliniciens sont des exemples vivants de ce que veulent devenir les étudiants. De plus, les modèles sont particulièrement importants lorsque les buts poursuivis sont très abstraits ou complexes et qu'ils peuvent difficilement être compris par les étudiants à partir des descriptions textuel les ${ }^{11,42}$.

La formation clinique est une période prolongée et soute nue d'immersion dans la culture professionnelle médicale: c'est à la foisl'endroit et le moment pendant lequel leprocessus detransformation professionnelle s'opère. Bowen et Carline ${ }^{43}$ soulignent que l'apprentissage est favorisé lorsque les étudiants et les résidents peuvent réciproquement échanger au sujet de leurs compréhensions et surtout lorsqu'ils peuvent observer des modèles de rôle experts et s'inspirer de leurs actions \& de leurs réflexions. Dans une enquête menée par Brownwell et Côté ${ }^{44}$ au cours de l'année académique 1998-1999 auprès de tous les résidents seniors ( $n: 533$ ) de deux facultés de médecine québécoises, les répondants ( $n$ : $258(48 \%)$ ) indiquaient que l'observation des modèles de rôleau cours de la résidence est la principale stratégie d'apprentissage du professionnalisme. Dans l'environnement pédagogique de l'enseignement clinique, les enseignants cliniciens ne sont pas les seuls à agir comme modèles de rôle auprès des externes et résidents. En effet, dans une étude au sujet des résidents, Wilkerson et al. ${ }^{45}$ rapportaient égal ement que le modèle de rôle était la modalitépédagogique utilisée le plus fréquemment par ceux-ci durant la tournée quotidienne. L'importance et l'impact potentiels du modèle de rôle par les enseignants pourraient donc être multipliés si on tenait également compte de l'effet additionnel de l'enseignement fait par les résidents. On peut cependant sinterroger sur les possibilités de distorsions successives si les modèles de rôle en amont ne sont pas optimaux.

Plusieurs travaux démontrent l'influence des modèles de rôle sur le choix de carrière ${ }^{11,46,47}$. Les étudiants identifient précocement des modèles de rôle, à un moment qui peut clairement jouer sur leur choix professionnel ${ }^{48}$.

\section{La définition du modèle de rôle}

$N$ ous avons été surpris de ne pas trouver, dans les écrits recensés, de définition reconnue de modèle de rôle. Seuls 
les auteurs suivants ont indiqué, avec plus ou moins de précision, ce qu'ils entendaient par ce terme. Wright ${ }^{49}$, sinspirant du dictionnaire Webster, propose cette définition des modèles de rôle : «A person considered as a standard of excellence to be imitated». Tiberius et al. ${ }^{50}$ proposent une définition similaire : «Someone whom learners perceived as worthy of emulatings. M audsley ${ }^{41}$ et Tiberius et al. ${ }^{50}$ élaborent davantage en apportant les nuances et distinctions suivantes concernant respectivement le modèle de rôle et le mentor. Le modèle de rôle se fait de façon consciente ou inconsciente ; il influence un ou plusieurs étudiants ; il n'existe pas nécessairement de relation formelleavec l'étudiant et la durée du contact avec celui-ci est variable. Par opposition, le mentor exerce son influence de façon intentionnelle et est engagé avec un ou un nombre limité d'étudiants dans une relation d'encadrement formelle et prolongée.

Dans le Dictionnaire actuel de l'éducation ${ }^{51}$, le terme « modèle de rôle » n'est pas explicité en tant que tel, les termes «modèle» et «rôle» étant abordés séparément. Le modèle y est défini commel' «idéal à atteindre par l'imitation d'un être ou par référence à un ensemble de caractéristiques à acquérir pour sapprocher d'un état de perfection : guide d'agir et de penser » et le rôle comme une «fonction assigné à quelqu'un, [un] comportement qu'il est convenu d'attendre de sa part ».

Prenant acte de l'absence de définition précise recensée dans la littérature, prenant en compte néanmoins les apports antérieurs des auteurs qui ont exploré la question et l'enrichissant des résultats d'entrevues semi-structurées que nous avons menés auprès d'enseignants cliniciens reconnus par leurs pairs, nous proposons la définition suivante du modèle de rôle en enseignement dinique : un modèle de rôle en médecine est un médecin qui, dans le contexte de son exercice professionnel, influence l'apprentissage des externes et des résidents avec qui il est en contact.

Cette définition permet de rendre compte dela variété des influences exercées par les modèles de rôle dans la pratique réelle, que ce soit en termes de direction (positive ou souhaitée vs négative ou non souhaitée) ou en termes de magnitude (négligeable vs significative).

\section{Les caractéristiques des bons modèles de rôle}

Les bons modèles de rôle sont ceux qui exercent sur l'apprentissage des étudiants une influence à la fois significative et conforme aux compétences finales visées. Pour que les modèles de rôle aient l'impact pédagogique recherché, encore faut-il que les étudiants les identifient et choisissent des'en inspirer. La perception des apprenants devient dès lors essentielle à considérer dans la description des modèles de rôle.

L'identification des caractéristiques des modèles de rôle a fait l'objet de plusieurs enquêtes ces dernières années, à la fois auprès d'étudiants, de résidents et d'enseignants cliniciens eux-mêmes. II semble que les perceptions des étudiants et des enseignants soient assez similaires. L'ensemble de ces sources converge vers l'identification de trois grandes catégories de caractéristiques des bons modèles de rôle: a) l'expertise clinique, qui est essentielle mais non suffisante ; $b$ ) les habiletés d'enseignant et c) les qualités personnelles. Le tableau 1 présentel'ensemble des caractéristiques regroupées sous ces trois catégories.

L'expertise clinique est rapportée par l'ensemble des auteurs comme étant une des caractéristiques essentielles des bons modèles de rôle ${ }^{49,52 \cdot 56}$. Cette expertise comporte la capacité à développer un raisonnement clinique performant dans les situations habituelles mais également dans les situations difficiles. Elle comprend également, pour les disciplines chirurgicales, des habiletés techniques expertes, une confiance et une capacité de prise de décision en contexte de procédures chirurgicales ${ }^{57}$. Elle inclue également la capacité de communiquer et d'interagir efficacement avec les patients et leurs familles.

Les habiletés d'enseignant font égal ement partie des caractéristiques rapportées des bons modèles de rôle $e^{49,52-55,57}$. Les bons modèles de rôleont une approche de l'enseignement davantage centrée sur l'apprenant ; ils impliquent activement les étudiants et ce de façon non menaçante ; ils communiquent efficacement avec les étudiants et leur donnent un feedback spécifiqueet en profondeur ; ils sont habiles pour expliquer les sujets difficiles ; enfin, ils portent une attention particulière à la relation médecinpatient dans leur enseignement.

Les qualités personnelles constituent la troisième catégorie de caractéristiques décrite chez les bons modèles de rôle ${ }^{49,52 \cdot 56,58}$. D ans cette catégorie, nous retenons particulièrement l'intégrité, la compassion, le souci des autres, l'enthousiasme par rapport à la profession médicale et à I'enseignement et l'engagement vers l'excellence et le développement. Une étude récente de Wright et Carrese ${ }^{54}$ concernant les caractéristiques des bons modèles de rôle est particulièrement intéressante. Par une approche qual itative utilisant des entrevues semi-structurées auprès de 29 enseignants cliniciens identifiés comme excellents 


\section{Références}

\begin{tabular}{|c|c|}
\hline \multicolumn{2}{|c|}{$\begin{array}{l}\text { Tableau } 1 \text { : } \\
\text { C aractéristiques des bons modèles de rôle }\end{array}$} \\
\hline Expertise clinique & Qualités personnelles \\
\hline $\begin{array}{l}\text { - Raisonnement clinique et prise de décision } \\
\text { - Communication efficace avec les patients }\end{array}$ & \multirow{3}{*}{$\begin{array}{l}\text { - Compassion } \\
\text { - Intégrité, honnêteté } \\
\text { - H abilités interpersonnelles et souci des autres } \\
\text { - Enthousiasme pour l'enseignement } \\
\text { et la médecine } \\
\text { - Engagement vers l'excellence } \\
\text { et le développement } \\
\text { - Sens de l'humour }\end{array}$} \\
\hline H abiletés d'enseignant & \\
\hline $\begin{array}{l}\text { - Approche centrée sur l'étudiant } \\
\text { - Communication efficace avec les étudiants }\end{array}$ & \\
\hline \multicolumn{2}{|c|}{$\begin{array}{l}\text { Les caractéristiques des bons modèes de rôle ont fait l'obje d'éudes descriptives. Ce tableau résume les caractéristiques } \\
\text { répertoriées et regroupéés sous trois catégories: l'expertise dinique, les habiletés d'enseg gnement e lesqual ités personnelles }\end{array}$} \\
\hline
\end{tabular}

modèles de rôles par les résidents, les auteurs confirment les caractéristiques précédemment décrites et explorent deux nouvelles dimensions de l'exercice du modèle de rôle : la conscience d'agir comme modèle de rôle d'une part, les barrières à l'exercice de ce rôle d'autre part.

Tous les professeurs questionnés disent exercer consciemment leur modèle de rôle lorsqu'ils sont en interaction avec les étudiants et ils font même des efforts additionnels dans les situations cliniques plus difficiles. D e plus, ils adoptent une approche plus « active » du modèle de rôle et ils deviennent explicites à propos des habiletés et comportements démontrés. Cette conscientisation est vue comme un levier pour maximiser l'impact du modèle sur l'apprentissage ; elle permet d'exploiter le modèle de rôle comme opportunité d'enseignement des dimensions de la médecine qui sont moins souvent formellement abordées et explicitées. Par ailleurs, l'impatience, la rigidité, le dogmatisme, l'introversion et la surcharge de travail - lorsqu'il y a de multiples tâches simultanées à assurer, par exemple - sont les barrières identifiées par ces enseignants à l'exercice optimal du modèle de rôle. Au terme de leur article, Wright et Carrese ${ }^{54}$ proposent un modèle conceptuel de l'exercice du modèle de rôle dans lequel ils intègrent l'expertise clinique, la conscientisation, les habiletés d'enseignant, les qualités personnelles et les barrières (figure 3 ). De plus, le fait que les étudiants puissent être en contact avec différents modèles de rôlesemblenécessaire. L'exercice de la médecine s'exprime de façon très variée selon les disciplines et les styles des praticiens. Bien que ces derniers partagent des savoirs normatifs communs, leurs savoirs pratiques ou leur art - variables selon les contextes et les personnes - colorent d'une façon singulière leur pratique. Pour que les étudiants développent progressivement leur propre «personnalité » de praticien, ils doivent être exposés et doivent se confronter à de multiples modèles d'exercice ${ }^{11,54}$. Encore faut-il que les enseignants puissent explicitement distinguer les différents savoirs - respectivement normatifs et pratiques - qu'ils utilisent dans leur exercice quotidien, en illustrant comment ils les sollicitent et les articulent. En contrepartie, nous pouvons nous interroger sur l'importance, au sein du corps enseignant, d'avoir des modèles de rôles qui reflètent les diverses populations d'apprenants en terme de sexe et de minorités ethniques. II semble, selon W right et C arrese ${ }^{59}$, que les similitudes entre les modèles et les apprenants puissent en faciliter l'impact.

\section{Les limites actuelles des modèles de rôle}

II serait naif de prétendre que l'ensemble des enseignants cliniciens possède toutes les caractéristiques d'excellence décrites par Wright et Carrese ${ }^{54}$. D ans une étude précé dente du même auteur, les résidents n'identifiaient que la moitié de leurs enseignants cliniciens comme étant de bons modèles de rôle ${ }^{49}$. Skeff et $M$ utha ${ }^{60}$ qui signaient 
l'éditorial accompagnant l'article de Wright se sont d'ailleurs plutôt interrogés à propos de l'autre moitié des professeurs non retenus par les résidents : «D o less than half of medical teachers have the skills and behaviors of res pected physicians? Do learners recognize the value of the professional roles of less than half of faculty members? Do less than half of clinical teachers currently work in an environment that fosters mastery and demonstration of the characteristics of excellent physicians? There may be truth in each of these hypotheses».

Déà en 1974, Rezler ${ }^{61}$, dans une recension des écrits concernant le changement d'attitudes des étudiants au cours de leur formation médicale, rapportait que l'environnement favorise l'émergence d'un certain cynisme de la part des étudiants. Dans une enquête plus récente auprès de 515 externes des quatre facultés de médecine québécoi ses, $40 \%$ des 319 répondants disaient ne pas percevoir chez leurs professeurs cliniciens des habiletés et attitudes humanistes dans leurs relations avec les patients et les étudiants ${ }^{62}$. Les auteurs questionnaient l'adéquation des modèles de rôle rencontrés pendant l'externat pour l'acquisition par les étudiants des aptitudes de «soignant ». La limite la plus évidente dans l'exercice des modèles de rôle est probablement son caractère tacite ou implicite ${ }^{42}$. Ces modèles de rôle «silencieux » exercent leur rôle de clinicien et sont observés par les étudiants mais ils n'explicitent ni n'articulent à l'intention de ceux-ci ni les raisons, ni les justifications, ni les explications sur lesquelles ils basent leurs actions. II est probable que la majorité du modèle de rôle exercé collectivement par les enseignants cliniciens est plutôt de type silencieux, implicite ou tacite. D ans une étude concernant le modèle de rôle et la communication médecin-patient, même lorsque qu'ils sont questionnés spécifiquement sur la relation médecinpatient, la plupart des enseignants avaient des difficultés à expliciter leurs actions et les savoirs sous-jacents ${ }^{63}$. Bien quel'impact au niveau de l'apprentissage puisse être positif, le modèle de rôle silencieux comporte des limites ou des risques bien identifiés ${ }^{42,64:}$

- une grande variété d'interprétations de la part de l'étudiant (bonnes ou mauvaises) est possible lorsqu'il n'y a pas explicitation de l'action observée ;

- une action particulière d'un modèle de rôle peut ne pas être universellement acceptée (variance, composante personnelle) ; les valeurs et justifications doivent être clarifiées pour l'étudiant ;

- un modèle tacite ne permet ni la réflexion, ni la remise en question, ni l'exercice de la métacognition.
Cette recension des écrits sur le modèle de rôle confirme d'abord l'importance de cette modalité pédagogique. Elle fournit également des données intéressantes quant à l'exercice optimal du modèle de rôle ainsi que des informationsfécondes sur les limites qu'il nousfaudratenter de repousser.

\section{Conclusions et perspectives}

Pour conclure, revenons d'abord aux deux questions initial ement posées : quelles sont les compétences du professeur clinicien expert? Q uelles sont les modalités pédagogiques à privilégier dans le cadre de la formation clinique?

En réponse à la première question, nous retenons que I'enseignant clinicien expert se caractérise par une double compétence, en l'occurrence la compétence clinique et la compétence pédagogique. La compétence clinique est multidimensionnelle et son explicitation par les enseignants, associée à la révélation des connaissances sous jacentes pour chacune des dimensions, est capitale pour son enseignement et pour son apprentissage par les étudiants. La compétence pédagogique est égal ement nécessaire. Elle comprend des domaines de connaissances spé cifiques en pédagogie et la capacité d'articuler ces domaines de connaissances avec les domaines de connais sances en médecine pour effectuer une démarche de raisonnement pédagogique particulière et centrée sur l'apprenant. Elle comprend également des stratégies métacognitives qui permettent de planifier l'enseignement, d'intervenir en choisissant des modalités efficaces et d'évaluer l'enseignement par la réflexion dans et après l'action. $N$ otre réponse à la deuxième question est la suivante : en enseignement clinique, le modèle de rôle et la supervision sont les deux modalités pédagogiques spécifiques qui permettent d'intégrer l'enseignement et l'apprentissage des différentes dimensions de la compétence clinique. Ces deux modalités sont centrées sur la résolution de problè me et sur la réalisation et la pratique de tâches professionnelles authentiques. Bien que ces deux modalités soient essentielles et complémentaires, l'impact du modèle de rôle semble déterminant. Les caractéristiques des bons modèles de rôle sont décrites assez clairement dans la littérature et lemodèle conceptuel de Wright ${ }^{54}$ (Figure 3) est particulièrement intéressant car il est cohérent avec la double compétence de l'enseignant clinicien expert déjà documentée. D ans les écrits actuels en éducation médicale, il semble y avoir convergence des discours concer- 


\section{Références}

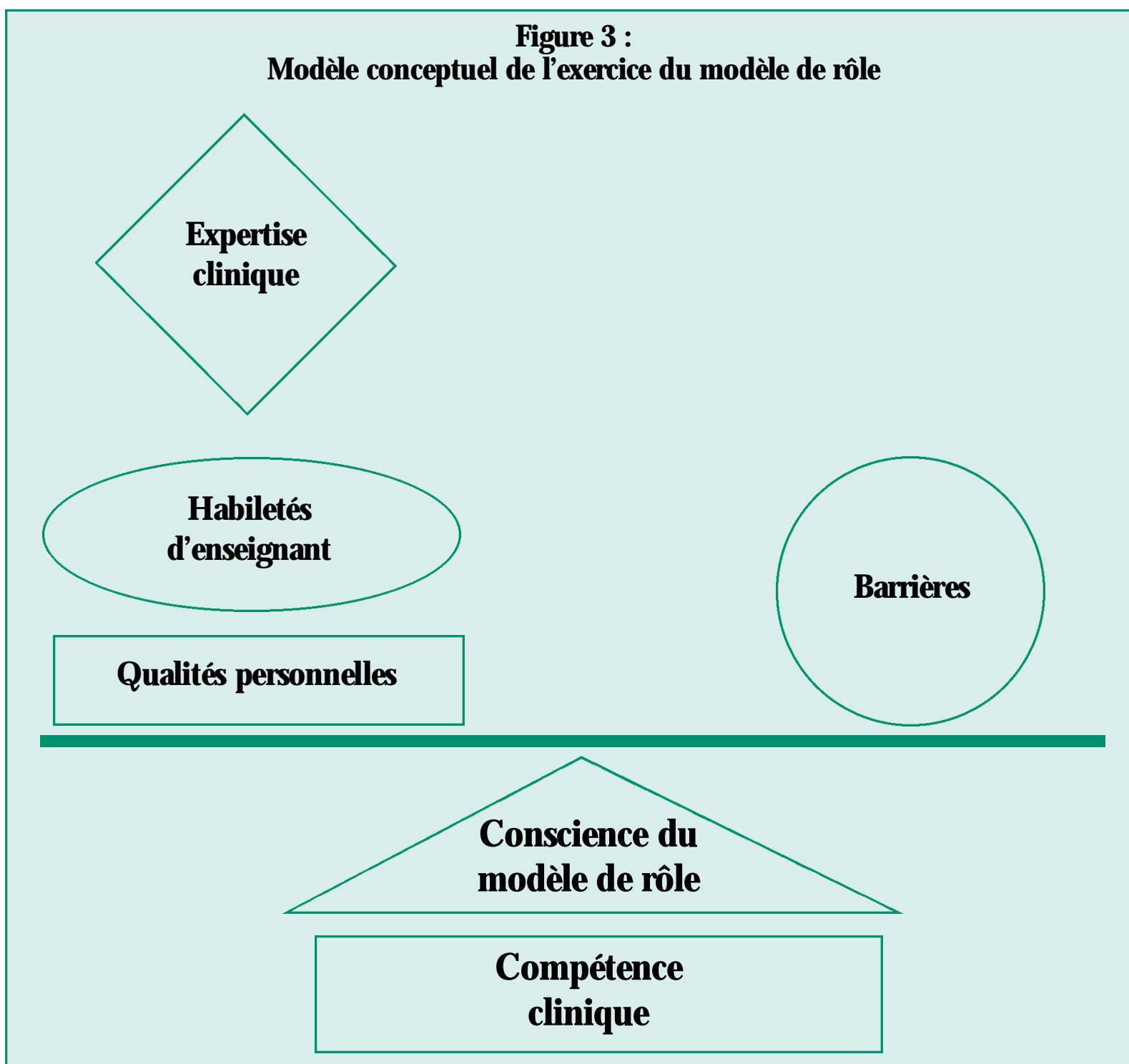

Figure illustrant un modèle conceptuel de l'exercice du modèle de rôle, dans lequel les barrières sont contrebalancées par des caractéristiques positives.

Référence : Wright 2002. Excellence in role model ling : insight and perspectives from the pros CM AJ $2002 ; 167(6): 638-43: p .640$ Traduction par l'auteur

Figure reproduite avec l'autorisation de l'Association médicale canadienne, en tant qu'éditeur du Journal de l'Association M édicale Canadienne.

nant l'importance du modèle de rôle comme modalité d'enseignement et d'apprentissage en enseignement clinique. L'optimisation des modèles de rôle figure donc clairement à l'agenda du perfectionnement pédagogique en enseignement clinique au cours des années à venir, comp- te tenu particulièrement des dimensions de la compétence clinique nouvellement explicitées et visées chez les étudiants diplômés ${ }^{10,11,41}$. «Good clinical teaching is concerned with providing role models for good practice, making good practice visible and explaining it to the trainees... Finding 
ways of assising dinicians to do this should be the basis of educational development for dinical teaching ${ }^{8}$

Bien qu'il y ait consensus sur la nécessité de ce perfectionnement pédagogique, les conclusions de la recherche se limitent au niveau des souhaits et desorientations. A notre connaissance, il n'existe aucun modèle opérationnel pour un tel perfectionnement pédagogique. Les données disponibles concernant les caractéristiques des bons modèles de rôle permettent cependant de croire que ceux-ci sont modifiables et que l'on pourrait vraisemblablement pallier les limites actuelles de ces modèles par la formation des enseignant ${ }^{49,54,60}$. Du modèle conceptuel de Wright ${ }^{54}$ concernant l'exercice optimal du modèle de rôle, deux caractéristiques peuvent être acquises ou modifiées : la conscience du modèle de rôle et les habiletés d'enseignant. Accroître la conscience du modèle de rôle et aider les enseignants dans leur démarche d'explicitation de la compétence clinique multidimensionnelle semble être une première piste intéressante pour le perfectionnement professoral. Une deuxième piste potentielle est de développer davantage les habiletés d'enseignant, d'une part, en proposant un cadre théorique de l'apprentissage et de l'enseignement centré sur l'apprenant et, d'autre part, en formant les professeurs à utiliser de façon optimale les deux modalités pédagogiques spécifiques du contexte de l'enseignement clinique que sont le modèle de rôle et la supervision.

\section{Contributions}

$M$ artine $C$ hamberland a effectué la recherche documentaire, procédé à l'analyse bibliographique et a rédigé les versions initiale et finale du manuscrit. $M$ artine $C$ hamberland et René $H$ ivon ont conjointement élaboré la synthèse conceptuelle. René Hivon a participé aux révisions intermédiaire et finale du manuscrit.

\section{Références}

1. Collège des médecins du Québec. Nouveaux défis professionnels pour le médecin des années 2000. Commission sur l'exercice de la médecine des années 2000. M ontréal, QC, 1998.

2. Collège Royal des médecins et chirurgiens du Canada. Compétences pour le nouveau millénaire: Rapport du groupe de travail sur les besoins sociétaux: ProM EDS 2000. Proje canadien d'éducation des médecins spécialistes. Royal college of Physicians \& Surgeons of Canada. Skillsfor the new millenium. Report of the societal needs working group. CanM EDS. 2000 project. 0 ttawa, Ont, 1996.

3. American Association of Medical Colleges (AAM C) Graduate Medical Education Core Curriculum. Core curriculum working group. Washington, 2000.

4. General Medical Council. Tomorrow's Doctors. Recommendations on undergraduate medical education. Première version 1993. Révision juillet 2002. London (UK), 2002.

5. Hafferty FW Franks R. The hidden curriculum, ethics teaching and the stucture of medical education. Acad M ed $1994 ; 69: 861-871$.

6. Hafferty FW. Beyond curriculum reform: confronting medicinés hidden curriculum. Acad M ed $1998 ; 73$ : 403-407.

7. Heskeh EA. Bagnall G, Buckley EG, Friedman M, Goodall E, H arden RM, Laidlaw JM, Leighton-Beck L, $M$ cKinglay $P, N$ ewton $R, O$ ughton $R$. A framework for developing excellence as a dinical educator. M ed Educ $2001 ; 35: 555-564$.

8. Prideaux $D$, Alexander $H$, Bower $A, D$ acre $J, H$ aist $S$, Jolly B, Norcini J, RobertsT, Rothman A, Rowe R, Tallet S. Clinical teaching: maintaining an educational rol efor doctors in the new health care environment. 9 th Cambridge conference on Clinical teaching. M ed Educ $2000 ; 34: 820-826$.

9. Parsell $G$, Bligh J. Recent perspectives on dinical teaching. M ed Educ 2001 ; 35 : 409-414.

10. H arden RM , CrosbyJ. AM EE Guide N o 20: The good teacher is more than a lecturer-the twelve roles of the teacher. M ed Teach 2000 ; 22 : 334-347.

11. Westberg J. Jason H . Chap 9 Serving as a role model. In: Collaborative Clinical Education: the foundations of effective Health Care Springer Series on Medical Education vol 16. New York (USA): Springer Publishing Company, 1993 : 155-165. 


\section{Références}

12. Irby D M. What clinical teachers need to know. Acad M ed $1994 ; 69$ : 333-342.

13. Irby D M . Three exemplary models of case based teaching. Acad M ed $1994 ; 69$ : 947-953.

14. Irby DM. How attending Physicians Make Instructional Decisions When Conducting Teaching Rounds. Acad M ed $1992 ; 67$ : 630-638.

15. Ullian JA, Bland CJ, Simpson DE. An alternative approach to defining the role of the clinical teacher. Acad M ed $1994 ; 69$ : 832-838.

16. Branch WT, Kroenke K, Levinson W. The dinicianeducator: present and future roles J Gen Intern M ed 1997 ; 12 (suppl)2: s1-4.

17. $\mathrm{H}$ iggs J, Jones $\mathrm{M}$. Clinical reasoning in the Health Professions, 2nd Ed. Oxford : ButterworthHeinemann, 2000.

18. Pate VL, Arocha JF, Kaufman DR. Expertise and tacit knowledge in medicine. In: Sternberg RJ. H orvath JA (eds). Tacit knowledge in Professions Practice. Researcher and Practitionner Perspectives M ahwah NJ, Lawrence Erlbaum Publishers, 1999 : 75-99.

19. Irby DM . Clinical teaching and the clinical teacher. J M ed Educ $1986 ; 61: 35-45$.

20. Shul man $L$. Those who understand : knowledge growth in teaching. Educational Researcher $1986 ; 15: 4-14$.

21. Shulman L. Knowledge and Teaching: Foundations of the new reform. H arvard Educ Rev $1987 ; 57: 1-22$.

22. Schmidt $H, N$ orman $G, B$ oshuizen H A. A Cognitive perspective on Medical Expertise: Theory and Implications. Acad M ed $1990 ; 65$ : 611-622.

23. Pinsky LE. I rby D M. "If at first you don't succeed": using failure to improve teaching. Acad M ed 1997 ; 72 : 973-976.

24. Pinsky LE, M onson D, Irby D M . H ow excellent teachers are made: reflecting on succes to improve teaching. Adv Health Sa Educ Theory Pract $1998 ; 3$ : 207 215.

25. Schön, DA. Educating the reflective practitioner: Toward a new design for teaching and learning in the professions. San Francisco (CA) : Jossey-Bass 1987.

26. Harden RM , CrosoyJR, DavisM H, Smith SR, D ollase $R$, Friedman Ben-David M, Ross N, Davies D, Hamilton J, Callahan D. O utcomebased education. AMEE Education Guide no 14. Dundee, Écosse AM EE, Centre for M edical Education, 1999.
27. Skeff KM, Stratos GA, M ygdal WK, DeWitt TG, $M$ anfred LM, Q uirk ME, Roberts KB, Greenberg LW. Clinical Teaching Improvement: Past and Future for Faculty D evelopment. Fam M ed $1997 ; 29$ : 252-257.

28. H offman Kimberly G, Donaldson Joe F. Contextual tensions of the clinical environnement and their influence on teaching and learning. M ed Educ 2004 ; $38: 448-454$.

29. K olb D . Experiential learning. Experienceas a source of learning and development. Englewood Cliffs (NJ) : Prentice-H all, 1984.

30. Schön, DA. The reflective practitioner: How professionals think in action. N ew York: Basic Books, 1983.

31. Brown JS, CollinsA, D uguid P. Situated cognition and the culture of learning. Educational Researcher 1989 ; $18: 32-42$.

32. Collins A, Brown JS, N ewman SE. Cognitive apprenticeship: Teaching the crafts of reading, writing and mathematics In: Remick LB (eds). Knowing, learning and instruction: Essays in honor of Robert Glaser. Hillsdale (N J): Lawrencw Erlbaum Associates, 1989 : 453-494.

33. Tardif J. Le Transfert des Apprentissages M ontréal (QC) : Les Editions LogiquesC anada, 1999.

34. Westberg J, Jason $\mathrm{H}$. Fostering reflection and Providing feedback: Helping Others Learn from Experience. Springer Series on Medical Education. New York, (NY) : Springer Publishing Company, 2001.

35. Lave J, Wenger E. Situated Learning: Legitimate peripheral participation. Cambridge (UK): Cambridge University Press, 1991.

36. Pratt $D D \&$ Associates at the University of British Columbia. Five perspective on teaching in adult and higher education. M alabar (FL) : Krieger, 1998.

37. Wooliscroft J. M edical Student Clinical education. In: Norman GR, Van der Vleuten CPM, N ewbleDI (eds). International $\mathrm{H}$ andbook of research in Medical Education Dordrecht: Kluwer Academic publishers, 2002: 365-380.

38. Whitcomb M E. The most serious challenge facing academic medicinés institutions. Acad M ed $2003 ; 78$ : 1201-1202.

39. Kenny N P, M ann KV, M acLoed $H$. Role modeling in Physicians' Professional Formation: Reconsidering an Essential but Untapped Educational Strategy. Acad M ed $2003 ; 78: 1203-1210$. 
40. Tosteson DC. Learning in M edicine N Engl J Med $1979 ; 301: 690-697$.

41. M audsley RF. Role Models and the Learning Environment : Essential Elements in Effective M edical education. Acad M ed $2001 ; 76: 432-434$.

42. Kenny N P, M ann KV. See one, D o one, Teach One: RoleM odelsand the Can M EDS Competencies Annals RCPSC $2001 ; 34: 435-438$.

43. Bowen JL, Carline J. Learning in the social Context of ambulatory careclinics Acad M ed 1997; 72 : 187-190.

44. Brownel AK, Côté $L$. Senior residentśviews on the meaning of professionalism and how they learn about it. Acad M ed $2001 ; 76$ : 734-737.

45. Wilkerson L, Lesky L, M edio FJ. The Resdent as Teacher duringWork Rounds J M ed Educ $1986 ; 61$ : 823-829.

46. Campos 0 utcalt D, Senf J, Watkins AJ, Bastacky S. The effects of M edical School Curricula, Faculty role models, and biomedical reserach support on choice of generalist physician careers : a Review and Quality assessment of the Literature Acad M ed 1995; 70 : 611-619.

47. Xu G, Rattner SL, Veloski JJ, Hojat M, Fields SK, Barzansky B. A National Study of the Factors Influencing $M$ en and Women Physicianśchoices of Primary care specialties Acad M ed $1995 ; 70$ : 398404.

48. Basco WT Jr, Reigart JR. When do medical students identify carer-influencing physician role modes? Acad M ed $2001 ; 76: 380-382$.

49. Wright $S$, Kern DE, Kolodner K, H oward D, Brancati FL. Attributes of excellent attending-physician role models. N Engl J M ed 1998 ; 339 : 1986-1993.

50. Tiberius RG, Sinai J, Flak EA. The Role of teacherlearner relationshipsin medical education. In: N orman GR, Van der Vleuten CPM, Newble DI (Eds). International $\mathrm{H}$ andbook of research in Medical Education. Dordrecht: Kluwer Academic publishers, 2002: 463-497.

51. Legendre R. Dictionnaire Actuel de l'Éducation. 2e Edition. M ontréal $(\mathrm{Q} C)$ : Guérin Editeur Limité, 1993.
52. Wright $\mathrm{S}$. Examining what reidents look for in their rolemodes. Acad M ed $1996 ; 71: 290-292$.

53. Wright $S$, Wong A, Newill $C$. Theimpact of rolemodes on medical sudents J Gen Intern M ed 1997 ; 12 : 53-56.

54. Wright SM, Carrese JA. Excellence in role modelling: insight and perspectives from the pros. CM AJ 2002 ; $167: 638-643$.

55. Ambrozy DM , Irby DM, Burack JH, Carline JD, Stritter FT. Role modes's perceptions of themselves and their influence on student's specialty choices. Acad M ed $1997 ; 72$ : 1119-1121.

56. Althouse LA, Stritter FT, Steiner BD. Attitudes and approaches of influential Role Modes in Clinical Education. Adv Health Sci Educ Theory Pract 1999 ; $4: 111-122$.

57. M aker VK, Curtis KD, D onnelly M B. Are you a surgical role model? Curr Surg $2004 ; 61: 111-115$.

58. Elzubeir M A, Rizk DE. I dentifying characteristics that students, interns and reidents look for in ther role models. M ed Educ $2001 ; 35$ : 272-277.

59. Wright SM, Carrese JA. Serving as a physician role model for a diversepopulation of medical learners. Acad M ed $2003 ; 78: 623-628$.

60. Skeff K, M utha S. Role modds- Guiding the future of M edicine. N Engl J M ed 1998 ; 339 : 2015-2017.

61. Rezler AG . AttitudeChanges D uring M edical School: A Review of the Literature. J Med Educ 1974; 49 : 1023-1030.

62. M aheux B, Beaudoin C, Berkson L. Côté $L$, $D$ esM archaisJE, Jean P. M edical faculty as humanistic and teachers: the perception of sudents at innovative and traditional medical schools M ed Educ 2000 ; 34 : 630-634.

63. Côté $L$, Leclère $H$. H ow clinical teachers perceive the doctor-patient relationship and themselves as role models. Acad M ed $2000 ; 75$ : 1117-11124.

64. Erde EL. The inadequacy of role models for educating medical sudents in ethics with some reflections on virtue theory. In: Thomasma D (ed) The influence of Edmund $D$. Pellegrino's philosophy of medicine Norwell: Kluwer Academic publishers. $1997:$ 31-34.

M anuscrit reçu le 10 janvier 2005 ; commentaires éditoriaux formulés aux auteurs le 30 mars 2005 ; accepté pour publication le 2 avril 2005 